\title{
Modelling persistent giardiasis and malnutrition
}

Persistent infection with Giardia lamblia alters mucosal structures in the gut and impairs host growth in a new mouse model, with concomitant malnutrition compounding these growth decrements, according to new research published in the Journal of Clinical Investigation.

G. lamblia is one of the most common enteric parasites infecting humans worldwide. Given that this infection can be recurrent or persistent and coincide with malnutrition, the long-term effects warrant investigation.

"Prior work included field studies, some of which associated giardiasis with linear childhood growth shortfalls," says author
Luther Bartelt. "We had previously demonstrated that malnutrition influenced disease outcomes following infection with Cryptosporidium and enteroaggregative Escherichia coli in mouse models," he adds, which gave the researchers the impetus to adapt this model to giardiasis.

Previous giardiasis mouse models used the trophozoite stage for challenge, but the researchers found that infection was transient in this scenario. By challenging mice with $G$. lamblia $\mathrm{H} 3$ cysts (the transmissable and infective form of Giardia in humans), they developed a mouse model of persistent giardiasis. Chronic inflammation, growth faltering and alterations in the gut mucosal

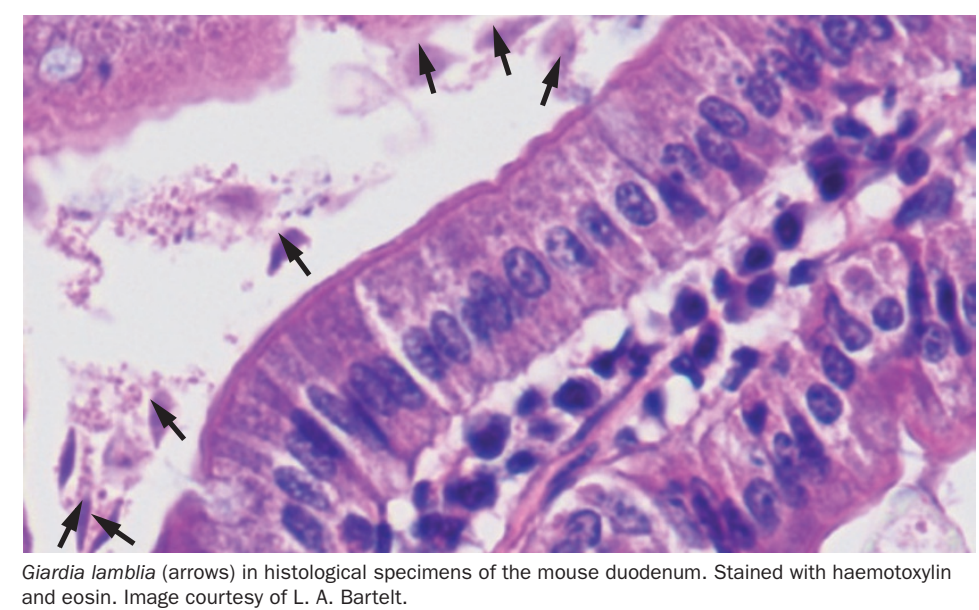

architecture (such as intestinal crypt hyperplasia) were observed, which mimicked some aspects of the disease in humans.

Furthermore, Bartelt et al. found that, in the context of persistent G. lamblia infection, malnourished mice (on a diet of only $2 \%$ protein) had further impaired growth compared with nourished mice (on a diet of $20 \%$ protein) as controls. Mice were weight-matched prior to the study.

The authors argue that their new model will enable further study to better understand the pathogenesis of chronic infection and also reveal mechanisms wherein enteropathogens and gut microbiota can influence host development. "Understanding the associations between enteropathogens and childhood development, and the pathogenesis of these microbes, could lead to novel therapies for overcoming the scourge of impaired growth and development that afflicts millions of children worldwide," reasons Bartelt.

Katrina Ray

Original article Bartelt, L. A. et al Persistent G. lamblia impairs growth in a murine malnutrition model. J. Clin. Invest. doi:10.1172/JCI67294 